\title{
Control and Synchronization of Chaos in RCL-Shunted Josephson Junction with Noise Disturbance Using Only One Controller Term
}

\author{
Di-Yi Chen, ${ }^{1,2}$ Wei-Li Zhao, ${ }^{1}$ Xiao-Yi Ma, ${ }^{1}$ and Run-Fan Zhang1 \\ ${ }^{1}$ Department of Electrical Engineering, Northwest AEF University, Shaanxi, Yangling 712100, China \\ ${ }^{2}$ College of Mechanical and Electric Engineering, Northwest AEF University, Shaanxi, \\ Yangling 712100, China \\ Correspondence should be addressed to Xiao-Yi Ma, ieee307@163.com
}

Received 27 March 2012; Accepted 26 May 2012

Academic Editor: Jinhu Lü

Copyright (c) 2012 Di-Yi Chen et al. This is an open access article distributed under the Creative Commons Attribution License, which permits unrestricted use, distribution, and reproduction in any medium, provided the original work is properly cited.

\begin{abstract}
This paper investigates the control and synchronization of the shunted nonlinear resistive-capacitive-inductance junction (RCLSJ) model under the condition of noise disturbance with only one single controller. Based on the sliding mode control method, the controller is designed to eliminate the chaotic behavior of Josephson junctions and realize the achievement of global asymptotic synchronization of coupled system. Numerical simulation results are presented to demonstrate the validity of the proposed method. The approach is simple and easy to implement and provides reference for chaos control and synchronization in relevant systems.
\end{abstract}

\section{Introduction}

Josephson junction, a strongly nonlinear device, has attracted considerable attention due to the advantage of ultra low noise, low power consumption, and high working frequency [1]. And thus different models were proposed as follows: the shunted linear resistive-capacitive junction (RCSJ) [2, 3], the shunted nonlinear resistive-capacitive junction (SNRCJ) [4], the shunted nonlinear resistive-capacitive-inductance junction (RCLSJ) [5] and the periodically modulated Josephson junction (PMJJ) [6,7].

The chaotic behavior of Josephson junctions has been widely investigated. Take vortex dynamics, for example, Josephson vortices in intrinsic Josephson junctions made of single crystalline $\mathrm{Bi}_{2} \mathrm{Sr}_{2} \mathrm{CaCu}_{2} \mathrm{O}_{8+\delta}$ [8], vortex dynamics in S-shaped Josephson junctions [9], vortex dynamics in Josephson junction arrays with magnetic flux noise measurements [10], dynamics of vortices in disordered Josephson junction arrays [11], vortex dynamics in Josephson 
junction arrays with percolative disorder [12]. At the same time, intrinsic Josephson junctions, formed in cuprate high-Tc superconductors, have won great attention. MachHida et al. [13] put forward microscopic theory for intrinsic Josephson junctions and established proper model equation describing the dynamic in intrinsic Josephson junctions. Kashiwaya et al. [14] studied the switching dynamics of $\mathrm{Bi}_{2} \mathrm{Sr}_{1.6} \mathrm{La}_{0.4} \mathrm{CuO}_{6+\delta}$ intrinsic Josephson junctions to show the effectiveness of the strong coupling between stacked junctions. Hayashi et al. [15] described the dynamics in a differential equation of intrinsic Josephson junctions under applied current.

However, it is essential to avoid all types of noise, chaos, and so forth, hence the need for chaos control and synchronization to make good use of the system. Motivated by important applications, researchers have put forward kinds of approaches to chaos control and synchronization, such as fuzzy control method [16], backstepping control method [17], feedback control method [18, 19], adaptive control method [20], OGY method [21], and sliding mode control method [22, 23]. As to Josephson junctions, in applications of parametric amplifiers [24], voltage standard [25], and superconducting quantum interference devices (SQUID) magnetometer [26], and so forth, chaos control and synchronization is of great help. Zhao and Wang [27] investigated the SNRCJ model, which contains two state variables and showed chaotic behavior with an external sinusoidal signal, and synchronized the model in two types of active siding control method as follows: strong restricting of the controller form and no superabundant restrictive assumption imposed for the controller. The study of the RCLSJ model which includes three state variables and exhibits chaotic dynamics with external DC bias only has been popular over years, and the model has been found to be more appropriate in high-frequency applications. In paper [28] a nonlinear active controller was proposed to synchronize the coupled RCLSJ model, and in paper [29] the RCLSJ model was synchronized via a variable structure controller with regard to uncertainties. Synchronization of superconducting junction arrays is also important. Gaifullin et al. [30] studied the synchronization of the stacked Josephson junctions array, fabricated by high-quality $\mathrm{Bi}_{2} \mathrm{Sr}_{2} \mathrm{CaCu}_{2} \mathrm{O}_{8+\delta}$ whiskers and crystals, making it possible to be applied in the fields as voltage standard or HTSC Josephson spectral detector. Filatrella and Pedersen [31] set up an intrinsic Josephson junction model of the highly anisotropic BSCCO type, and a cavity is used to enhance fluxon synchronization in a stacked junction, providing application for enhancing microwave radiation from BSCCO single crystals. In addition to the classic method of synchronization, in paper [32], cluster synchronization was introduced to realize the synchronization of coupled Josephson junction arrays. Recently, the chaos control and synchronization of the RCLSJ model has been achieved in paper $[33,34]$. In both papers, three control inputs were introduced to realize the achievement of the model control, and although the synchronization of the model was achieved with one control term, the control inputs did not consider the external disturbance in practical systems.

Consequently, the aim of this paper lies in the achievement of the control and synchronization of the RCLSJ model by means of sliding mode method with only one control term under the condition of noise disturbance. It is worth noting the control and synchronization aim considered in this paper can be both achieved with only a single control input. Furthermore, the single controller proposed in this paper regardless of the system's noise disturbance, which is of great significance in reducing the cost and complexity for controller implementation. This paper is organized as follows. Section 2 describes the model and discusses the dynamics behavior of the system, and Section 3 presents the sliding mode designs, together with numerical simulations. In Section 4, we summarize this work. 


\section{The Model of Josephson Junction System and Dynamics Analysis}

A standard form of the RCLSJ model [35] is proposed as

$$
\begin{gathered}
\frac{h}{4 \pi e} \frac{d \gamma}{d t}=V \\
C \frac{d V}{d t}+\frac{V}{R_{V}}+I_{c} \sin (\gamma)+I_{s}=I \\
L \frac{d I_{S}}{d t}+I_{S} R_{S}=V
\end{gathered}
$$

where $R_{S}$ and $L$ are the shunt resistance and inductance, $I_{S}$ the shunt current, $I$ the input DC bias. $I_{c}, C, R_{V}$ are critical current, capacitance, and resistance of the junction, respectively. The junction resistance $R_{V}$ is nonlinear, expressed as follows:

$$
R_{V}=\left\{\begin{array}{ll}
R_{N}, & |V|>V_{g} \\
R_{\mathrm{sg}}, & |V| \leq V_{g},
\end{array} \quad \text { with } V_{g}=\frac{2 \Delta}{e}\right.
$$

where $R_{N}$ and $R_{\mathrm{sg}}$ are the normal state resistance and energy gap resistance, $V_{g}$ the gap voltage. $\gamma$ and $V$ are the superconducting order parameter phase difference and the junction voltage.

For numerical simulation and analysis, the standard form of nonlinear nondimensional differential equation is got as

$$
\begin{aligned}
& \frac{d x_{1}}{d \tau}=x_{2}, \\
& \frac{d x_{2}}{d \tau}=\frac{1}{\beta_{c}}\left[i-g x_{2}-\sin \left(x_{1}\right)-x_{3}\right], \\
& \frac{d x_{3}}{d \tau}=\frac{1}{\beta_{L}}\left(x_{2}-x_{3}\right),
\end{aligned}
$$

with $x_{1}=\gamma, x_{2}=V / I_{c} R_{s}, x_{3}=I_{s} / I_{c}$.

Normalized time: $\tau=\omega_{c} t, \omega_{c}=4 \pi I_{c} R_{s} / h$.

Normalized current: $i=I / I_{c}$.

Normalized voltage: $v=V / I_{c} R_{s}$.

Other dimensionless parameters: $\beta_{c}=4 \pi I_{c} R_{S}^{2} C / h, \beta_{L}=4 \pi e I_{c} L / h, g=R_{S} / R_{V}$, where $\beta_{c}$ and $\beta_{L}$ are simplified capacitance and inductance, respectively. Further, the equation of dynamic system [33] can be obtained as

$$
\begin{aligned}
& \dot{x}_{1}=x_{2}, \\
& \dot{x}_{2}=a\left[d-c x_{2}-\sin \left(x_{1}\right)-x_{3}\right], \\
& \dot{x}_{3}=b\left(x_{2}-x_{3}\right) .
\end{aligned}
$$




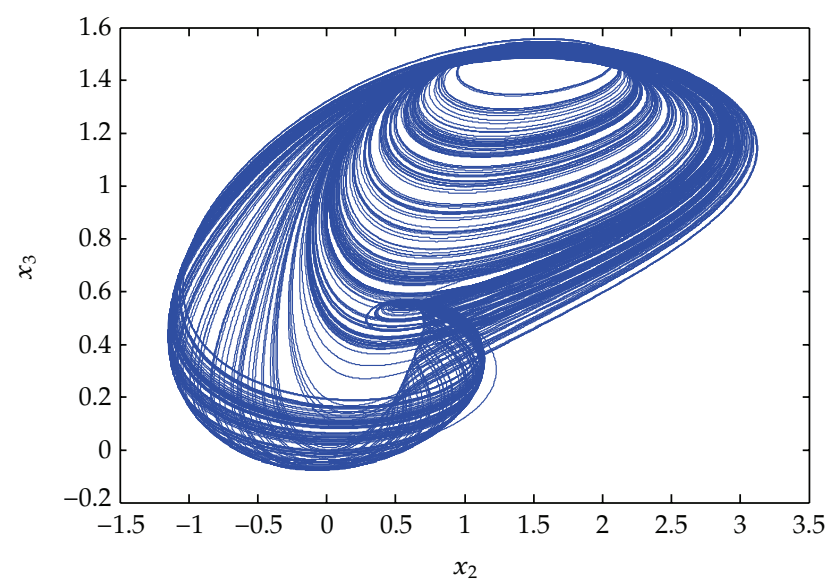

Figure 1: The $x_{2}-x_{3}$ two-dimensional phase diagram.

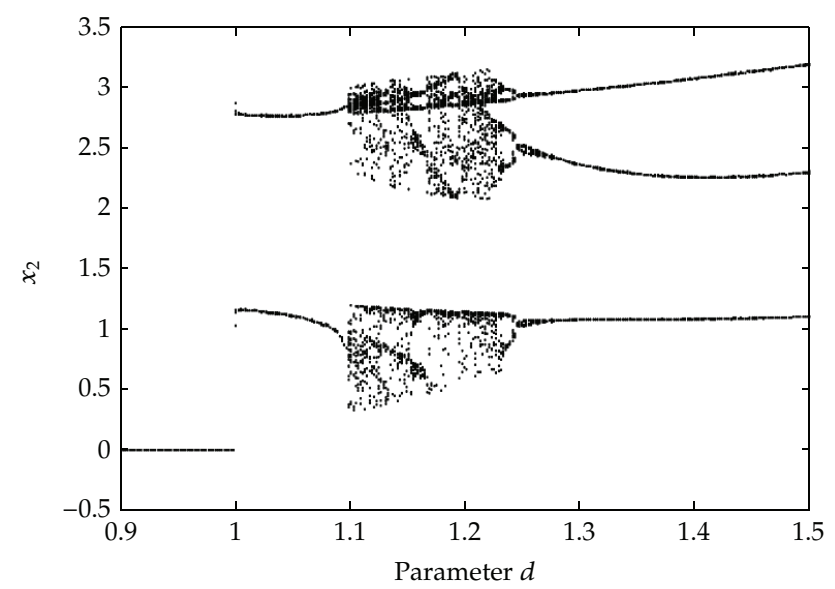

Figure 2: The bifurcation diagrams varying with $d$.

Let $\beta_{c}=0.707, \beta_{L}=2.68, g=0.0478, i=1.2$, that is, $a=1.4144, b=0.3731, c=0.0478$, $d=1.2$ and the initial condition $(0,0,0)$. The phase diagram is shown in Figure 1.

Bifurcation diagrams can be applied to discover the transitions between periodic motion and chaotic motion of the system with the system parameter varying. Figure 2 displays the bifurcation of the system (2.4) with respect to parameter $d$.

\section{Design of the Controller with Only One Control Term}

\subsection{Chaos Control in RCLSJ Model}

The controlled system is defined as follows:

$$
\begin{aligned}
& \dot{x}_{1}=x_{2}, \\
& \dot{x}_{2}=a\left[d-c x_{2}-\sin \left(x_{1}\right)-x_{3}\right]+\delta(t)+u, \\
& \dot{x}_{3}=b\left(x_{2}-x_{3}\right),
\end{aligned}
$$


where $u$ is the control input and $\delta(t)$ is the external disturbance of the system. Assume disturbance term $\delta(t)$ is bounded, that is, $|\delta(t)| \leq \alpha$ where $\alpha$ is positive constant.

The goal is to design a sliding controller and stabilize the system for any given initial condition. First of all, an adaptive switching surface is defined as

$$
S(t)=x_{2}(t)+\varphi(t),
$$

where $\varphi(t)$ is an adaptive function given by

$$
\dot{\varphi}=x_{1}+b x_{3}+a c x_{2}+\rho x_{2}, \quad \rho>0 .
$$

When the system operates in sliding mode, it satisfies the following equation:

$$
S(t)=\dot{S}(t)=0
$$

Therefore,

$$
\dot{S}(t)=\dot{x}_{2}(t)+\dot{\varphi}(t)=0 \Longrightarrow \dot{x}_{2}(t)=-\dot{\varphi}(t)=-x_{1}-b x_{3}-a c x_{2}-\rho x_{2}
$$

From (3.5) the following sliding mode dynamics can be obtained as

$$
\begin{gathered}
\dot{x}_{1}=x_{2} \\
\dot{x}_{2}=-x_{1}-b x_{3}-a c x_{2}-\rho x_{2} \\
\dot{x}_{3}=b\left(x_{2}-x_{3}\right) .
\end{gathered}
$$

In the following, the Lyapunov stability theory is used to analyze the stability of the sliding mode dynamics (3.6). The Lyapunov function is selected as $v(t)=0.5\left(x_{1}{ }^{2}+x_{2}{ }^{2}+x_{3}{ }^{2}\right)$, which leads to

$$
\begin{aligned}
\dot{v} & =x_{1} \dot{x}_{1}+x_{2} \dot{x}_{2}+x_{3} \dot{x}_{3}=x_{1} x_{2}+x_{2}\left(-x_{1}-b x_{3}-a c x_{2}-\rho x_{2}\right)+x_{3} b\left(x_{2}-x_{3}\right) \\
& =-(a c+\rho) x_{2}{ }^{2}-b x_{3}{ }^{2} \leq 0
\end{aligned}
$$

According to Lyapunov stability theory, it appears that the sliding motion on the sliding manifold is stable and ensures $\lim _{t \rightarrow \infty}\left\|\left[x_{1}, x_{2}, x_{3}\right]\right\|=0$ where $\|\cdot\|$ is the Euclidean norm of a vector.

The next step is to design a control scheme to drive the system trajectories onto the sliding mode $s=0$. The equivalent control law is obtained:

$$
u_{\mathrm{eq}}=-x_{1}-b x_{3}-\rho x_{2}-a\left(d-\sin x_{1}-x_{3}\right)-\delta(t)
$$

In general, the overall control signal has the following form:

$$
u=u_{\mathrm{eq}}+k_{s} \operatorname{sgn}(S)
$$

where $k_{s}$ is the switching gain. 
In practice, the system uncertainty, $\delta(t)$, is unknown. To overcome this, the equivalent control input is therefore modified to

$$
u=u_{\mathrm{eq}}+k_{s} \operatorname{sgn}(S)=-x_{1}-b x_{3}-\rho x_{2}-a\left(d-\sin x_{1}-x_{3}\right)+k_{s} \operatorname{sgn}(S) .
$$

Theorem 3.1. When $k_{s}<-\alpha$, the controller (3.10) can make the system (2.4) reach sliding mode $S=$ 0 and the trajectory of the system converge to the sliding surface $S(t)=0$ in a finite time.

Proof. The Lyapunov function of the system is constructed as $V=0.5 S^{2}$, and then its first derivative with respect to time is

$$
\begin{aligned}
\dot{V} & =S \dot{S}=S\left[a\left(d-c x_{2} \sin \left(x_{1}\right)-x_{3}\right)+x_{1}+b x_{3}+a c x_{2}+\rho x_{2}+\delta(t)+u\right] \\
& =S\left[\delta(t)+k_{s} \operatorname{sgn}(S)\right] \leq \alpha|S|+k_{s}|S|=\left(k_{s}+\alpha\right)|S|<0 .
\end{aligned}
$$

Thus the proof is achieved completely.

Without loss of generality, we choose the uncertainty term $\delta(t)=0.1 \cos (t)$, where $|\delta(t)| \leq \alpha=0.1$ and the initial conditions of the system $[0.5,0.8,0.4], k_{s}=-7$ and $\rho=7$. The system parameters $a=1.4144, b=0.3731, c=0.0478, d=1.2$ are specified for simulation.

The simulation results are modeled in MATLAB software by using fourth-order Runge-Kutta method and shown in the following figures. Figure 3 shows the time responses of the state variables of the uncontrolled system. Figure 4 shows the time domain charts of state variables of system when the control is active. Figure 5 shows the time-varying graph of sliding surface. Obviously, the simulation results presented confirm the validity of the proposed control.

\subsection{Synchronization of the Coupled RCLSJ Model}

For the advantage of ultralow noise, low power consumption and high working frequency for RCL, we were led to ask whether it would be possible to synchronize two different RCLSJ systems together.

The controlled system with noise perturbation is described as follows:

$$
\begin{aligned}
& \dot{y}_{1}=y_{2}, \\
& \dot{y}_{2}=a\left[d-c y_{2}-\sin \left(y_{1}\right)-y_{3}\right]+\delta(t)+U, \\
& \dot{y}_{3}=b\left(y_{2}-y_{3}\right),
\end{aligned}
$$

where $U$ is the control input and $\delta(t)$ is the external disturbance of the system. Assume disturbance term $\delta(t)$ is bounded, that is, $|\delta(t)| \leq \delta$ where $\delta$ is positive constant. 


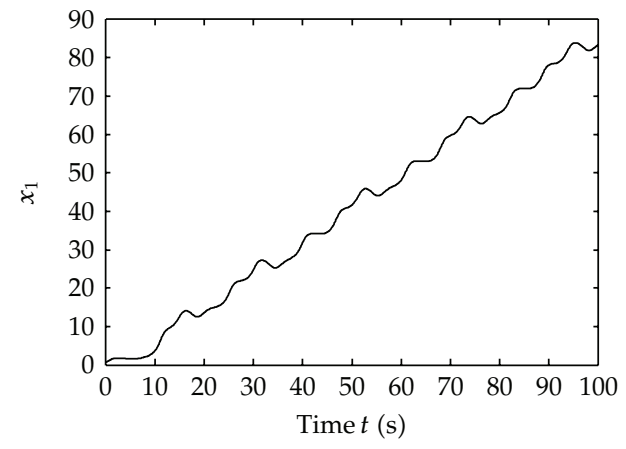

(a) $x_{1}-t$

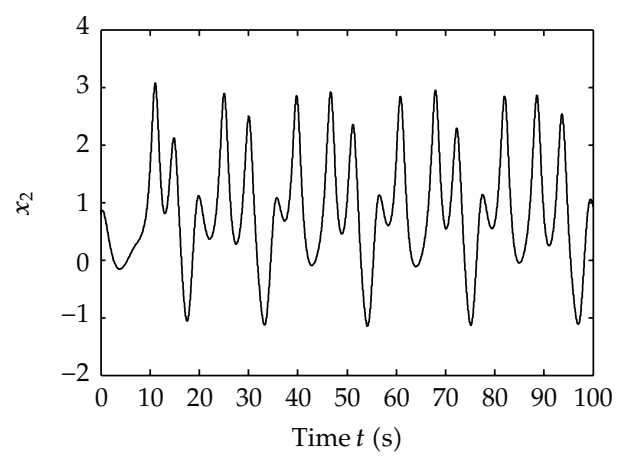

(b) $x_{2}-t$

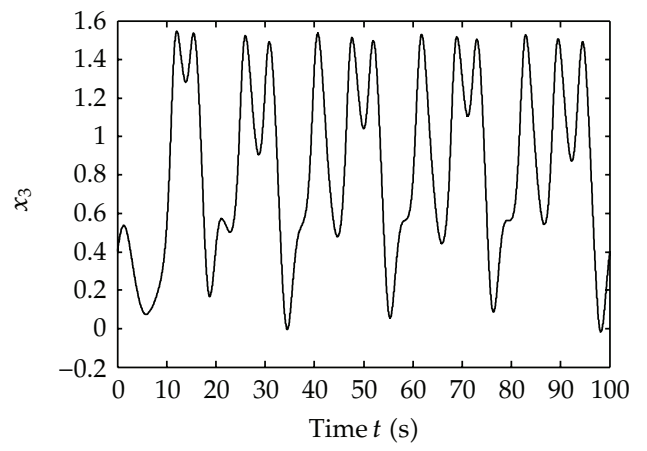

(c) $x_{3}-t$

Figure 3: The time domain charts of state variables without controller.

Define the error states of system as follows:

$$
\begin{aligned}
& e_{1}=y_{1}-x_{1}, \\
& e_{2}=y_{2}-x_{2}, \\
& e_{3}=y_{3}-x_{3} .
\end{aligned}
$$

The error dynamics system is obtained:

$$
\begin{aligned}
& \dot{e}_{1}=e_{2}, \\
& \dot{e}_{2}=-a\left(c e_{2}+e_{3}\right)+d(t)+U_{1}, \\
& \dot{e}_{3}=b\left(e_{2}-e_{3}\right),
\end{aligned}
$$

with $U_{1}=U-a\left(\sin y_{1}-\sin x_{1}\right)$. 


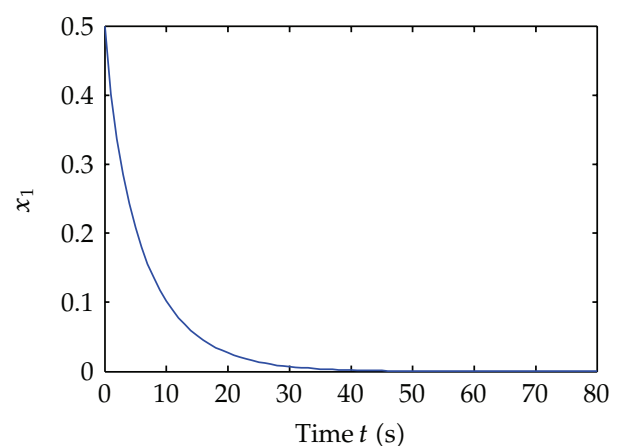

(a) $x_{1}-t$

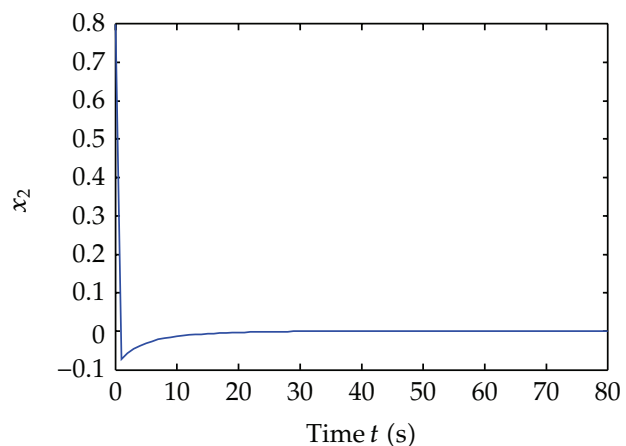

(b) $x_{2}-t$

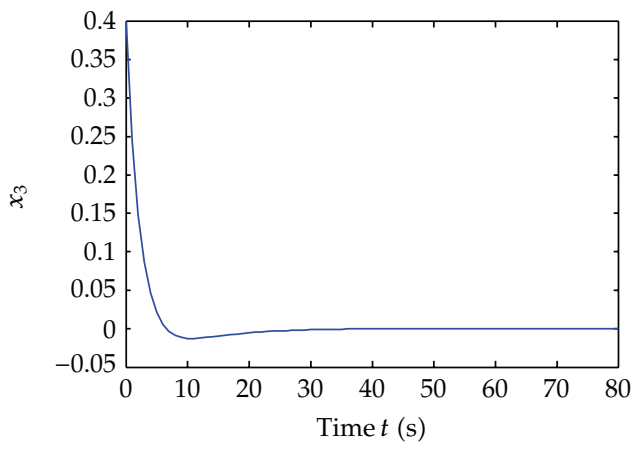

(c) $x_{3}-t$

Figure 4: The time domain charts of state variables when the control is applied.

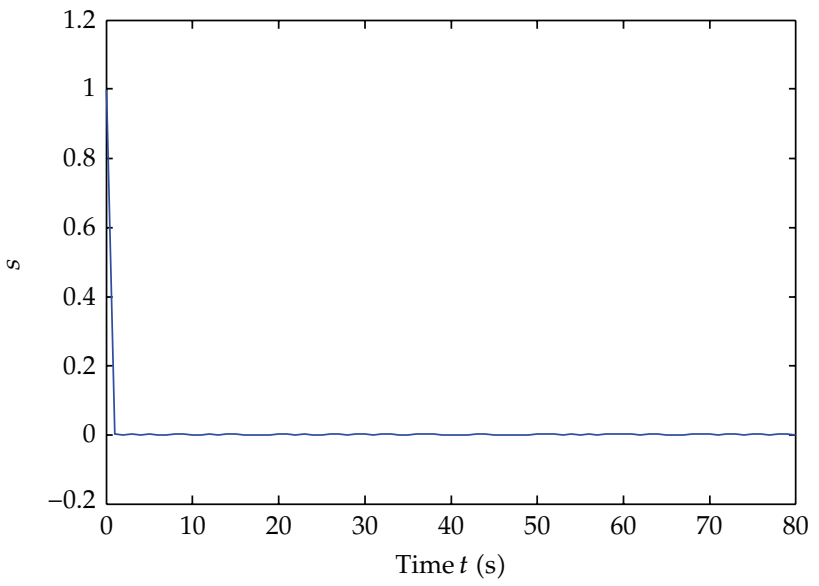

Figure 5: The time domain chart of sliding surface. 
We establish an extended system as follows:

$$
\begin{aligned}
& \dot{e}_{1}=e_{2}, \\
& \dot{e}_{2}=e_{4}, \\
& \dot{e}_{3}=b\left(e_{2}-e_{3}\right), \\
& \dot{e}_{4}=-a b\left(e_{2}-e_{3}\right)-a c e_{4}+\dot{d}(t)+\dot{U}_{1},
\end{aligned}
$$

and choose a sliding mode surface $s=0$, where

$$
s(t)=e_{4}+\int_{0}^{t}\left(c_{1} e_{1}+c_{2} e_{2}+c_{4} e_{4}\right) d \tau
$$

and $c_{1}, c_{2}, c_{4}$ are constants waiting for designing.

Theorem 3.2. If the controller $U_{1}$ is selected as

$$
U_{1}=\int_{0}^{t}\left[a c e_{4}+a b\left(e_{2}-e_{3}\right)-c_{1} e_{1}-c_{2} e_{2}-c_{4} e_{4}-k \operatorname{sgn}(s(t))\right] d \tau,
$$

where $s(t)$ is as in (3.16) and $k$ is constant satisfying $k>\delta+1$, then the states of the error system (3.13) will approach the sliding mode surface $s=0$ in a finite time.

Proof. The Lyapunov function of the system is selected as $V=s^{2}$; with the section of the sliding mode surface (3.16) and the controller (3.17), we have

$$
\begin{aligned}
\dot{V} & =2 s\left(\dot{e}_{4}+c_{1} e_{1}+c_{2} e_{2}+c_{4} e_{4}\right) \\
& =2 s\left[-a c e_{4}-a b\left(e_{2}-e_{3}\right)+c_{1} e_{1}+c_{2} e_{2}+c_{4} e_{4}-\delta(t)+\dot{u}_{1}\right] \\
& =2 s[-\dot{d}(t)-k \operatorname{sgn}(s)] \leq 2(|-\dot{d}(t)|-k)|s| \leq-2|s|=-2 V^{1 / 2}
\end{aligned}
$$

which implies that, $V^{1 / 2}(t) \leq V^{1 / 2}(0)-t, t \in\left[0, t_{s}\right)$ and $V(t)=0$ when $t \geq t_{s}$ where $t_{s}=V^{1 / 2}(0)$. The proof is finished as desired.

Next we analyze the dynamics of the error system on the sliding manifold. On the sliding manifold $s=0$ the error system (3.15) reads

$$
\begin{aligned}
& \dot{e}_{1}=e_{2}, \\
& \dot{e}_{2}=e_{4}, \\
& \dot{e}_{3}=b\left(e_{2}-e_{3}\right), \\
& \dot{e}_{4}=-c_{1} e_{1}-c_{2} e_{2}-c_{4} e_{4}+\delta(t) .
\end{aligned}
$$


Thus,

$$
\left(\begin{array}{c}
e_{1} \\
e_{2} \\
e_{4}
\end{array}\right)=e^{A t}\left[\left(\begin{array}{c}
e_{1}(0) \\
e_{2}(0) \\
e_{4}(0)
\end{array}\right)+\int_{0}^{t} e^{-A s}\left(\begin{array}{c}
0 \\
0 \\
\dot{d}(t)
\end{array}\right) d s\right],
$$

where

$$
A=\left(\begin{array}{ccc}
0 & 1 & 0 \\
0 & 0 & 1 \\
-c_{1} & -c_{2} & -c_{4}
\end{array}\right)
$$

Its characteristic polynomial is

$$
f(\lambda)=|\lambda I-A|=\lambda^{3}+c_{4} \lambda^{2}+c_{2} \lambda+c_{1}
$$

According to Routh-Hurwitz theorem, we know that the real parts of its all characteristic roots are negative if and only if

$$
\Delta_{1}=c_{4}>0, \quad \Delta_{2}=c_{4} c_{2}-c_{1}>0, \quad \Delta_{3}=c_{1}\left(c_{4} c_{2}-c_{1}\right)>0 .
$$

Therefore, there exists positive constants $a_{1}$ and $b_{1}$ such that $\left|e^{A t} x\right| \leq a_{1} e^{-b_{1} t}|x|$ for every $x \in R^{3}$ and $t \geq 0$. Thus,

$$
\left|e_{i}\right| \leq a_{1} e^{-b_{1} t} \max _{i=1,2,4}\left|e_{i}(0)\right|+\frac{a_{1} \delta}{b_{1}}, \quad i=1,2,4
$$

Additionally, (3.19) implies that

$$
e_{3}=e^{-b t}\left(e_{3}(0)+\int_{0}^{t} e^{b t} b e_{2} d t\right)
$$

Therefore,

$$
\left|e_{3}\right| \leq e^{-b t}\left(\left|e_{3}(0)\right|+\int_{0}^{t} e^{b t}\left|b e_{2}\right| d t\right) .
$$

Obviously, we can get the following theorem.

Theorem 3.3. If the controller $U$ is selected as $U=U_{1}+a\left(\sin y_{1}-\sin x_{1}\right)$, where $U_{1}=\int_{0}^{t}\left[\right.$ ace $_{4}+$ $\left.a b\left(e_{2}-e_{3}\right)-c_{1} e_{1}-c_{2} e_{2}-c_{4} e_{4}-k \operatorname{sgn}(s(t))\right] d \tau, c_{1}, c_{2}, c_{4}$ are constants satisfying (3.23) and $k>\delta+1$, there exists a constant $M$ such that the controlled chaotic system with noise perturbation (3.12) is synchronous with the system (2.4) with ultimate error bound $M \delta$, that is:

$$
\varlimsup_{t \rightarrow \infty}\left|y_{1}-x_{1}\right| \leq M \delta, \quad \varlimsup_{t \rightarrow \infty}\left|y_{2}-x_{2}\right| \leq M \delta, \quad \varlimsup_{t \rightarrow \infty}\left|y_{3}-x_{3}\right| \leq M \delta .
$$




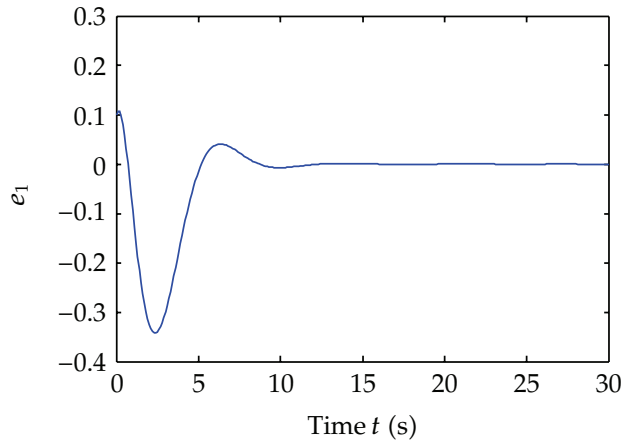

(a) $e_{1}-t$

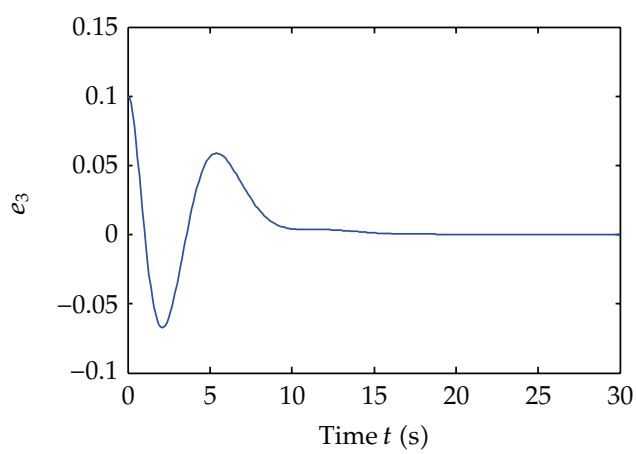

(c) $e_{3}-t$

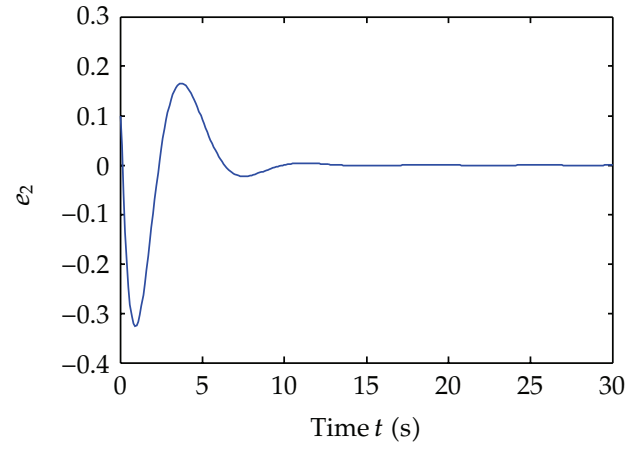

(b) $e_{2}-t$

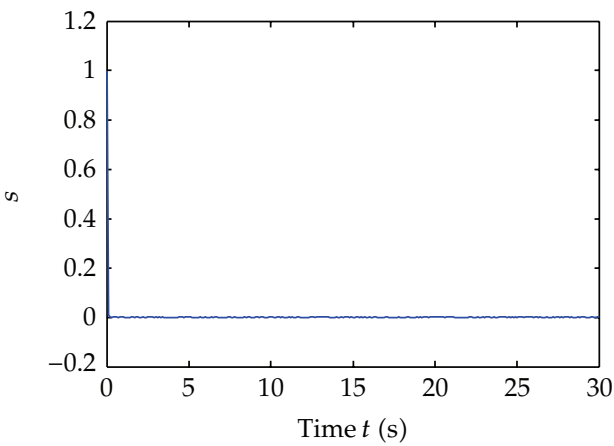

(d) $s-t$

Figure 6: The time response of the error system and sliding mode surface.

Without loss of generality, we choose the uncertainty term $\delta(t)=0.1 \cos (t)$, where $|\delta(t)| \leq \delta=1$. In the following numerical simulations, the parameters of the system are $a=$ $1.4144, b=0.3731, c=0.0478, d=1.2$; the initial conditions of the system (2.4) and the system (3.12) are $\left[\begin{array}{lll}0.4 & 0.5 & 0.7\end{array}\right]^{T}$ and $\left[\begin{array}{lll}0.5 & 0.6 & 0.8\end{array}\right]^{T}$, respectively. The constants in the sliding mode are selected $c_{1}=1, c_{2}=2, c_{4}=2$. And the constant in the sliding mode controller is selected as $k=10$.

Numerical simulations are presented to demonstrate the effectiveness of the proposed method. Figure 6 displays the time response of the synchronization errors defined in (3.13) and sliding mode surface defined in (3.16) when the control signal has been activated. It is very clear that the error dynamics converge asymptotically to zero as soon as the control is activated. The numerical simulation verifies the theoretical analysis.

\section{Conclusions and Discussion}

We have investigated the control and synchronization of the RCLSJ model via sliding mode method. In the sliding mode design, the single controller is constructed in the case of noise disturbance for the chaos control of the junction, and numerical simulation results are employed to verify the effectiveness of the control scheme. And it is same with the chaos synchronization of the junction. In practical system, with noise disturbance considered, the control scheme is of significant importance due to its simple and effective execution as well as good robustness. 
Here, another interesting remark is that chaos control and chaos synchronization could be realized by using the same method. Comparing the chaos control and synchronization, we can get the conclusion that chaos control and chaos synchronization are the same with each other in essence. In other words, chaos control is special case of chaos synchronization, and it is considered to achieve synchronization with $\mathrm{O}(0,0,0)$. Moreover, applying this control method to multiscroll chaotic systems [36-39] is our future work.

\section{Acknowledgments}

This wok was supported by the scientific research foundation of National Natural Science Foundation (51109180) and the "111" Project from the Ministry of Education of P.R. of China and the State Administration of Foreign Experts Affairs of P.R. of China (B12007) and the National Science and Technology Supporting Plan from the Ministry of Science and Technology of P.R. of China (2011BAD29B08).

\section{References}

[1] T. G. Zhou, D. C. Wang, F. L. Liu, L. Fang, X. J. Zhao, and S. L. Yan, "Simulation of chaos in different models of Josephson junctions," Journal of System Simulation, vol. 22, no. 3, pp. 666-669, 2010.

[2] C. R. Nayak and V. C. Kuriakose, "Dynamics of coupled Josephson junctions under the influence of applied fields," Physics Letters A, vol. 365, no. 4, pp. 284-289, 2007.

[3] S. Das, S. Datta, and D. Sahdev, "Mode-locking, hysteresis and chaos in coupled Josephson junctions," Physica D, vol. 101, no. 3-4, pp. 333-345, 1997.

[4] E. Kurt and M. Canturk, "Chaotic dynamics of resistively coupled DC-driven distinct Josephson junctions and the effects of circuit parameters," Physica D, vol. 238, no. 22, pp. 2229-2237, 2009.

[5] X. S. Yang and Q. D. Li, "A computer-assisted proof of chaos in Josephson junctions," Chaos, Solitons $\mathcal{E}$ Fractals, vol. 27, no. 1, pp. 25-30, 2006.

[6] T. Kawaguchi, "Directed transport and complex dynamics of vortices in a Josephson junction network driven by modulated currents," Physica C, vol. 470, no. 20, pp. 1133-1136, 2010.

[7] S. Al-Khawaja, "Chaotic dynamics of underdamped Josephson junctions in a ratchet potential driven by a quasiperiodic external modulation," Physica C, vol. 420, no. 1-2, pp. 30-36, 2005.

[8] K. Kadowaki, I. Kakeya, T. Yamamoto, T. Yamazaki, M. Kohri, and Y. Kubo, “Dynamical properties of Josephson vortices in mesoscopic intrinsic Josephson junctions in single crystalline $\mathrm{Bi}_{2} \mathrm{Sr}_{2} \mathrm{CaCu}_{2}$ $\mathrm{O}_{8+\delta}$, , Physica C, vol. 437-438, pp. 111-117, 2006.

[9] P. D. Shaju and V. C. Kuriakose, "Vortex dynamics in S-shaped Josephson junctions," Physica C, vol. 434, no. 1, pp. 25-30, 2006.

[10] S. Candia, Ch. Leemann, S. Mouaziz, and P. Martinoli, “Investigation of vortex dynamics in Josephson junction arrays with magnetic flux noise measurements," Physica C, vol. 369, no. 1-4, pp. 309-312, 2002.

[11] T. Kawaguchi, "Depinning mechanism and driven dynamics of vortices in disordered Josephson junction arrays," Physica C, vol. 392-396, part 1, pp. 364-368, 2003.

[12] J. Affolter, A. Eichenberger, S. Rosse, P. Scheuzger, C. Leemann, and P. Martinoli, "Phase and vortex dynamics in Josephson junction arrays with percolative disorder," Physica B, vol. 280, no. 1-4, pp. 241-242, 2000.

[13] M. MacHida, T. Koyama, A. Tanaka, and M. Tachiki, "Theory of the superconducting phase and charge dynamics in intrinsic Josephson-junction systems: microscopic foundation for longitudinal Josephson plasma and phenomenological dynamical equations," Physica C, vol. 331, no. 1, pp. 85-96, 2000.

[14] H. Kashiwaya, T. Matsumoto, H. Shibata et al., "Switching dynamics and MQT in Bi2201 intrinsic Josephson junctions," Physica C, vol. 469, no. 15-20, pp. 1593-1595, 2009. 
[15] M. Hayashi, M. Suzuki, J. Onuki, and H. Ebisawa, "Nonlinear dynamics of intrinsic Josephson junctions under an applied current," Physica C, vol. 463-465, pp. 993-996, 2007.

[16] J. M. Dias and A. Dourado, "A self-organizing fuzzy controller with a fixed maximum number of rules and an adaptive similarity factor," Fuzzy Sets and Systems, vol. 103, no. 1, pp. 27-48, 1999.

[17] D. Y. Chen, L. Shi, H. T. Chen, and X. Y. Ma, "Analysis and control of a hyperchaotic system with only one nonlinear term," Nonlinear Dynamics, vol. 67, no. 3, pp. 1745-1752, 2012.

[18] J. Ma, Q. Y. Wang, W. Y. Jin et al., “Control chaos in the Hindmarsh-Rose neuron by using intermittent feedback with one variable," Chinese Physics Letters, vol. 25, no. 10, pp. 3582-3585, 2008.

[19] M. Rafikov and J. M. Balthazar, "On control and synchronization in chaotic and hyperchaotic systems via linear feedback control," Communications in Nonlinear Science and Numerical Simulation, vol. 13, no. 7, pp. 1246-1255, 2008.

[20] Z. Ruo-Xun and Y. Shi-Ping, "Chaos in fractional-order generalized lorenz system and its synchronization circuit simulation," Chinese Physics B, vol. 18, no. 8, pp. 3295-3303, 2009.

[21] A. S. de Paula and M. A. Savi, "A multiparameter chaos control method based on OGY approach," Chaos, Solitons E Fractals, vol. 40, no. 3, pp. 1376-1390, 2009.

[22] D. Y. Chen, W. L. Zhao, X. Y. Ma, and R. F. Zhang, "No-chattering sliding mode control chaos in Hindmarsh-Rose neurons with uncertain parameters," Computers \& Mathematics with Applications, vol. 61, no. 10, pp. 3161-3171, 2011.

[23] D. Y. Chen, R. F. Zhang, X. Y. Ma et al., "Chaotic synchronization and anti-synchronization for a novel class of multiple chaotic systems via a sliding mode control scheme," Nonlinear Dynamics, vol. 69, no. 1-2, pp. 35-55, 2011.

[24] B. Yurke, R. Movshovich, P. G. Kaminsky et al., "Vacuum-noise squeezing at microwave frequencies using Josephson-parametric amplifier," Physica B, vol. 169, no. 1-4, pp. 432-435, 1991.

[25] F. Müller, H. Schulze, R. Behr, J. Kohlmann, and J. Niemeyer, "The Nb-Al technology at PTB-a common base for different types of Josephson voltage standards," Physica C, vol. 354, no. 1-4, pp. 66-70, 2001.

[26] E. Il'ichev, G. S. Krivoy, and R. P. J. IJsselsteijn, "Low frequency noise of high- $T_{c}$ radio-frequency SQUIDs based on grain boundary Josephson junctions," Physica C, vol. 377, no. 4, pp. 516-520, 2002.

[27] Y. Zhao and W. Wang, "Chaos synchronization in a Josephson junction system via active sliding mode control," Chaos, Solitons E Fractals, vol. 41, no. 1, pp. 60-66, 2009.

[28] A. Ucar, K. E. Lonngren, and E. W. Bai, “Chaos synchronization in RCL-shunted Josephson junction via active control," Chaos, Solitons E Fractals, vol. 31, no. 1, pp. 105-111, 2007.

[29] J. J. Yan, C. F. Huang, and J. S. Lin, "Robust synchronization of chaotic behavior in unidirectional coupled RCLSJ models subject to uncertainties," Nonlinear Analysis. Real World Applications, vol. 10, no. 5, pp. 3091-3097, 2009.

[30] M. B. Gaifullin, K. Hirata, S. Ooi, S. Savel'ev, Yu. I. Latyshev, and T. Mochiku, “Synchronization in stacked array of the Josephson junctions in $\mathrm{Bi}_{2} \mathrm{Sr}_{2} \mathrm{CaCu}_{2} \mathrm{O}_{8+\delta}$," Physica C, vol. 468, no. 15-20, pp. 18961898, 2008.

[31] G. Filatrella and N. F. Pedersen, "Synchronization of intrinsic Josephson junctions to a cavity," Physica C, vol. 408-410, no. 1-4, pp. 560-561, 2004.

[32] W. X. Qin and G. Chen, "Coupling schemes for cluster synchronization in coupled Josephson equations," Physica D, vol. 197, no. 3-4, pp. 375-391, 2004.

[33] U. E. Vincent, A. Ucar, J. A. Laoye, and S. O. Kareem, "Control and synchronization of chaos in RCL-shunted Josephson junction using backstepping design," Physica C, vol. 468, no. 5, pp. 374-382, 2008.

[34] A. N. Njah, K. S. Ojo, G. A. Adebayo, and A. O. Obawole, "Generalized control and synchronization of chaos in RCL-shunted Josephson junction using backstepping design," Physica C, vol. 470, no. 1314, pp. 558-564, 2010.

[35] C. B. Whan, C. J. Lobb, and M. G. Forrester, "Effect of inductance in externally shunted Josephson tunnel junctions," Journal of Applied Physics, vol. 77, no. 1, pp. 382-389, 1995.

[36] J. Lü, S. Yu, H. Leung, and G. Chen, "Experimental verification of multidirectional multiscroll chaotic attractors," IEEE Transactions on Circuits and Systems I, vol. 53, no. 1, pp. 149-165, 2006.

[37] S. Yu, J. Lü, X. Yu, and G. Chen, "Design and implementation of grid multiwing hyperchaotic lorenz system family via switching control and constructing super-heteroclinic loops," IEEE Transactions on Circuits and Systems I, vol. 59, no. 5, pp. 1015-1028, 2012. 
[38] J. Lü and G. Chen, "Generating multiscroll chaotic attractors: theories, methods and applications," International Journal of Bifurcation and Chaos in Applied Sciences and Engineering, vol. 16, no. 4, pp. 775858, 2006.

[39] J. Lü, F. Han, X. Yu, and G. Chen, "Generating 3-D multi-scroll chaotic attractors: a hysteresis series switching method," Automatica, vol. 40, no. 10, pp. 1677-1687, 2004. 


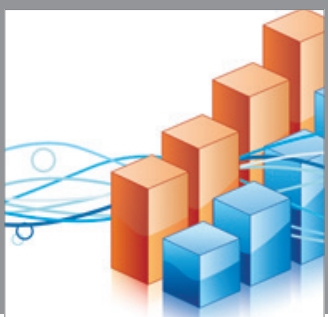

Advances in

Operations Research

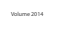

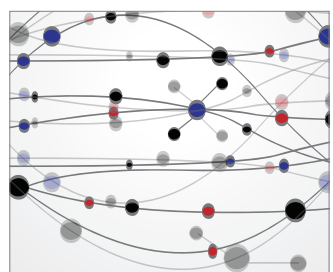

\section{The Scientific} World Journal
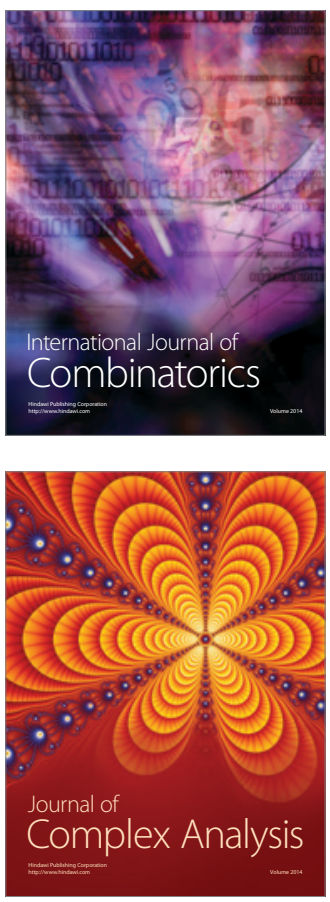

International Journal of

Mathematics and

Mathematical

Sciences
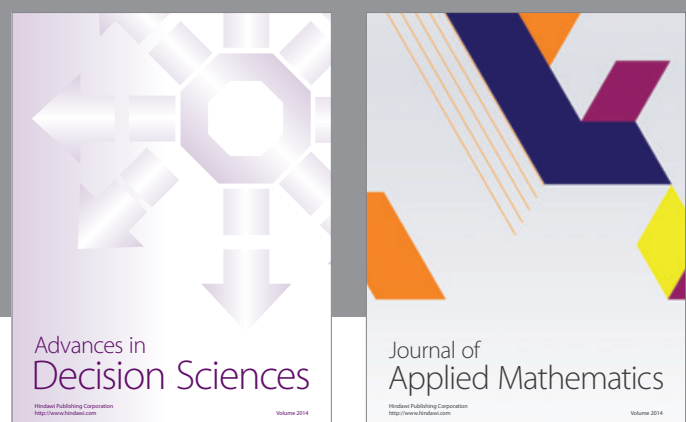

Journal of

Applied Mathematics
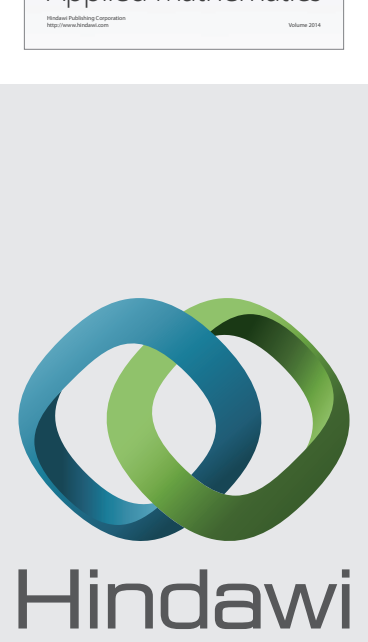

Submit your manuscripts at http://www.hindawi.com
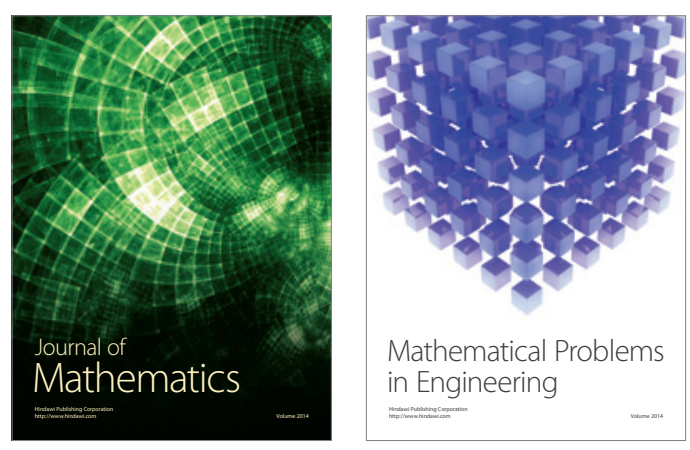

Mathematical Problems in Engineering
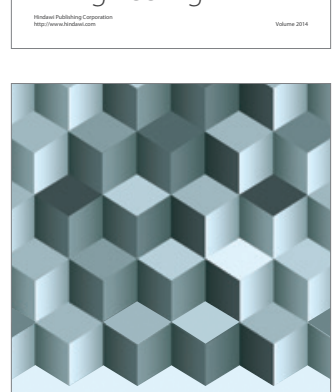

Journal of

Function Spaces
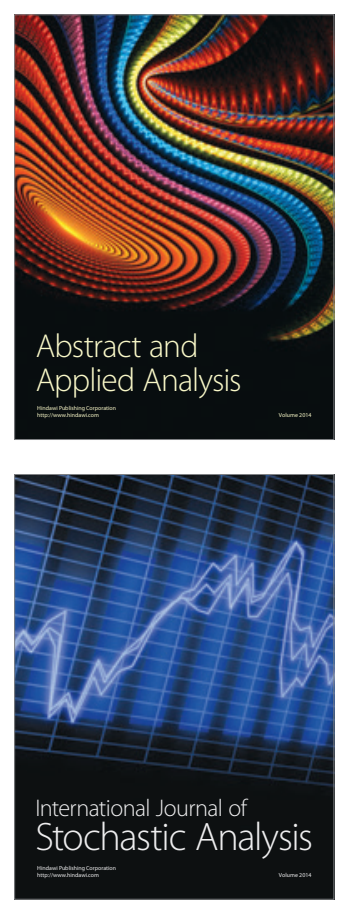

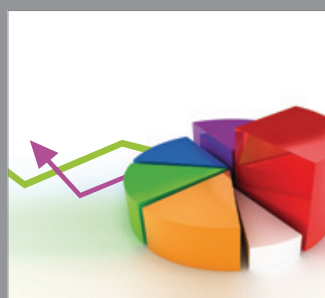

ournal of

Probability and Statistics

Promensencen
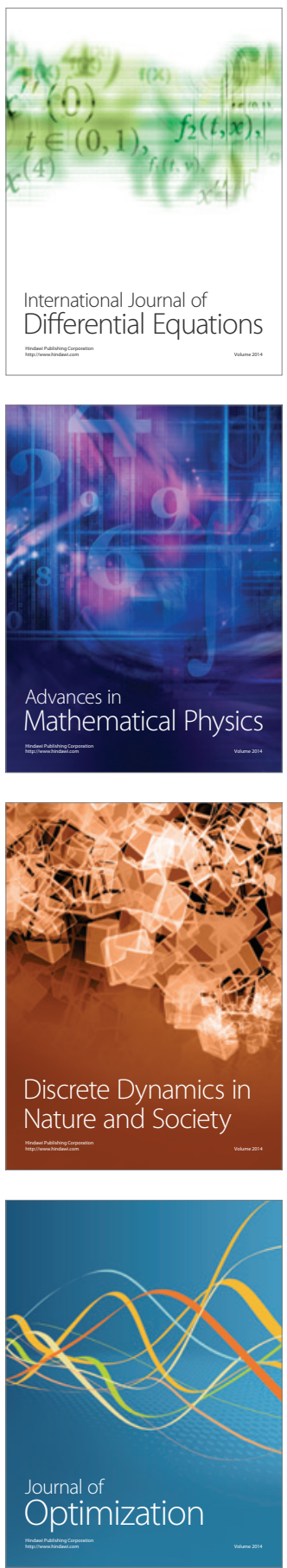